\title{
Attention Augmented Convolutional Transformer for Tabular Time-series
}

\author{
Sharath M Shankaranarayana ${ }^{1}$ and Davor Runje ${ }^{1,2}$
}

\begin{abstract}
Time-series classification is one of the most frequently performed tasks in industrial data science, and one of the most widely used data representation in the industrial setting is tabular representation. In this work, we propose a novel scalable architecture for learning representations from tabular time-series data and subsequently performing downstream tasks such as time-series classification. The representation learning framework is end-to-end, akin to bidirectional encoder representations from transformers (BERT) in language modeling, however, we introduce novel masking technique suitable for pretraining of time-series data. Additionally, we also use one-dimensional convolutions augmented with transformers and explore their effectiveness, since the time-series datasets lend themselves naturally for one-dimensional convolutions. We also propose a novel timestamp embedding technique, which helps in handling both periodic cycles at different time granularity levels, and aperiodic trends present in the timeseries data. Our proposed model is end-to-end and can handle both categorical and continuous valued inputs, and does not require any quantization or encoding of continuous features.
\end{abstract}

\section{INTRODUCTION}

Industrial entities in domains such as finance, telecommunication, and healthcare usually log a large amount of data of their customers or patients. The data is typically in the form of events data, capturing interactions their users have with different entities. The events could be specific to the respective domains, for example, financial institutions log all financial transactions made by their customers, telecommunication companies log all the interactions of the individual customers, national healthcare systems keep logs of all visits and diagnoses patients had in different healthcare institutions, etc. These kinds of data mostly employ tabular representation. A multivariate time-series data represented in tabular form is often referred to as dynamic tabular data [1] or tabular time-series. These datasets are rich for performing knowledge discovery, doing analytics, and also building predictive models using machine learning (ML) techniques. Even though there exists a large amount of data, the data often remains unexplored due to various inherent difficulties (such as unstructuredness, noise, sparse and missing values). Because of the complexities, usually only a subset of the data is employed for building ML models. Thus, a large amount of potentially rich and relevant data is unused for ML modeling. Moreover, machine learning using such data is frequently performed by first extracting hand-crafted or engineered features and later building task-specific machine learning models. This engineering of the features is known to be one of the biggest challenges in industrial machine

\footnotetext{
1 Airt Research, Zagreb, Croatia

2 Algebra University College, Zagreb, Croatia
}

learning, since it requires domain knowledge and timeconsuming experimentation for every different use case. Additionally, it has limited scalability because models cannot be automatically created and maintained. Another important issue is that most machine learning models require data to be in the form of fixed-length vectors or sequences of vectors. This is not straightforward and is difficult to obtain for such large time-series datasets. To this end, we propose a framework for learning vector representations from large time-series data. Our framework can later employ these learned representations on downstream tasks such as timeseries classification.

Learning numerical vector representations (embeddings), or representation learning, is one the major areas of research, specifically in the natural language processing (NLP) domain. In the seminal work called word2vec [2], vector representations of words are learnt from huge quantities of text. In word2vec, each word is mapped to a $d$-dimensional vector such that semantically similar words have geometrically closer vectors. This is achieved by predicting either the context words appearing in a window around a given target word (skip-gram model), or the target word given the context (called continuous bag of words or CBOW model). In this model, it is assumed that the words appearing frequently in similar contexts share statistical properties and thus this model leverages word co-occurrence statistics.

NLP has since then seen massive improvements in terms of results by building upon embeddings-related works. For example, the work [3] proposed a transfer learning scheme for text classification and thus heralded a new era by making the transfer learning in NLP on par with the transfer learning tasks in computer vision. Another recent work [4] proposed contextualized word representations, as opposed to static word representations.

The current state-of-the-art techniques in NLP learn vector representations of words using transformers and attention mechanism [5] on large datasets with the task of reconstructing an input text with some of the words in it randomly masked [6].

Upon seeing the immense gains of employing models with the attention mechanism and transformers in NLP, there have been a few works employing them for tabular data. In the work [7], the authors propose a network called TabNet, which is a transformer based model for selfsupervised learning for tabular data. They report significant gains in prediction accuracy when the labelled data is sparse, however, the work does not learn any sort of embeddings inherently and translates the use of transformer architecture 


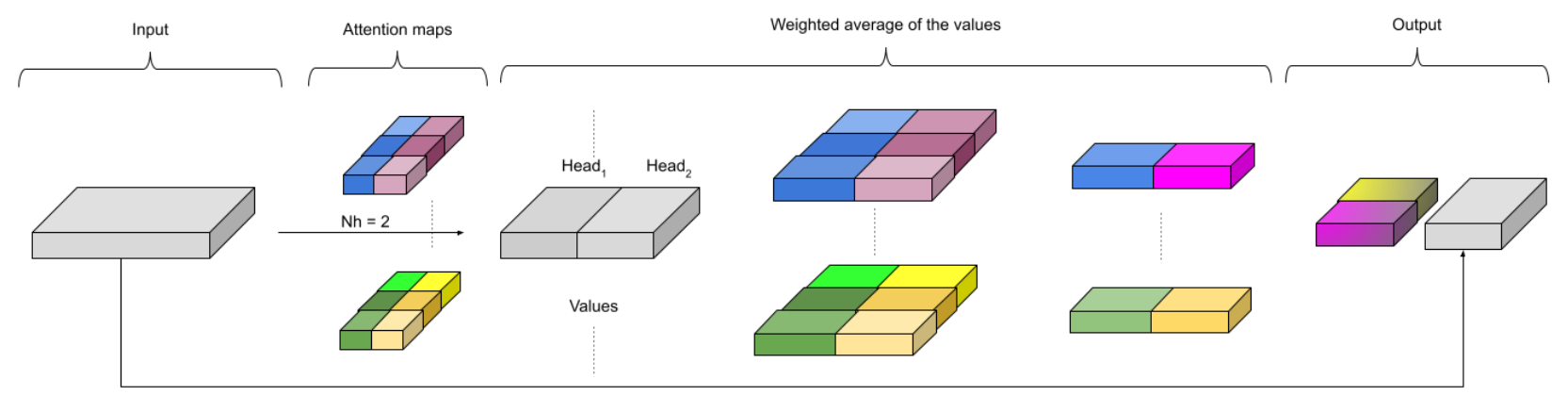

Fig. 1. Attention-augmented convolution: For each temporal location, $N_{h}$ attention maps over the image are computed from queries and keys. These attention maps are used to compute $N_{h}$ weighted averages of the values $V$. The results are then concatenated, reshaped to match the original input's dimensions and mixed with a pointwise convolution. Multi-head attention is applied in parallel to a standard convolution operation and the outputs are concatenated.

from NLP domain to tabular domain. Another similar very recent work [8] proposes similar use of transformers for the tabular data. In this work, although the authors do intend to learn embeddings, it is only for encoding categorical features present in the tabular data. Whereas in our work, we learn the embeddings for specific agents by contextualizing the interactions between the various agents. Thus, the embeddings we obtain are very informative, unlike broad encoding of categorical values. Most recently, the authors in [9] proposed the use of BERT architecture modified for time-series anomaly detection task.

To the best of our knowledge, the closest work to ours is TabBERT proposed in the work [1], in which the authors employ hierarchical BERT [10], [11] to first perform pretraining on time-series data and then later employ the embeddings on downstream tasks. Although similar, our proposed work differs in several aspects:

- We do not employ hierarchical BERT but single encoder layer consisting of transformers, and make use of embeddings to transform raw inputs in order to be fed to BERT, making its computational cost significantly smaller.

- We propose a masking technique specifically suited for time-series data resulting in higher performance of the model.

- TabBERT requires input data to be already encoded as categorical values, whereas our proposed framework can handle both discrete and continuous inputs.

Another relevant work to ours is [12], where the authors propose transformers for time-series representation learning, but the pretraining loss function employed is mean squared error loss for both continuous and discrete features, whereas in our method we apply classification loss for discrete type features and regression loss for floating features. To the best of our knowledge, ours is the first work that employs attention augmented convolutions [13] (as a part of BERT layer) for time-series data and also the first work that proposes timestamp embedding block.

In summary, the main contributions of our paper are as follows:

- We propose a novel BERT framework employing attention augmented convolutions for time-series tabular data TabAConvBERT.

- We propose a masking scheme that is better suitable for time-series tabular data.

- We propose a novel timestamp embedding block along with positional encoding. This timestamp embedding block helps in handling of both periodic cycles at different time granularity levels and aperiodic trends.

- The proposed framework can handle both discrete and continuous input types.

\section{Methodology}

\section{A. Attention Augmented Convolution}

One-dimensional (1-D) convolutions have long been employed in time-series tasks and have shown reasonably good results [14]. In computer vision, employing attention mechanisms along with convolutional neural networks have given significant improvements in various vision tasks [15], [16], [17], [18]. Recently, the authors of the work [13] proposed attention augmented convolutional networks that can jointly attend to both spatial and feature subspaces, in contrast to previous convolutional attention mechanisms, which either perform only channel-wise reweighing [15], [16] or perform reweighing of both channels and spatial positions independently[17], [18]. In this work, we propose a 1-D convolution version of attention augmented convolutions [13]. Similar to [13], the proposed attention augmented convolution

1) is equivariant to translation, and

2) can readily operate on inputs of different temporal dimensions.

As shown in [13], if we consider an original convolution operator with kernel size $k, F_{\text {in }}$ input filters, and $F_{\text {out }}$ output filters, the corresponding attention augmented convolution can simply be written as:

$$
A A \operatorname{Conv}(X)=\operatorname{Concat}[\operatorname{Conv}(X), M H A(X)]
$$




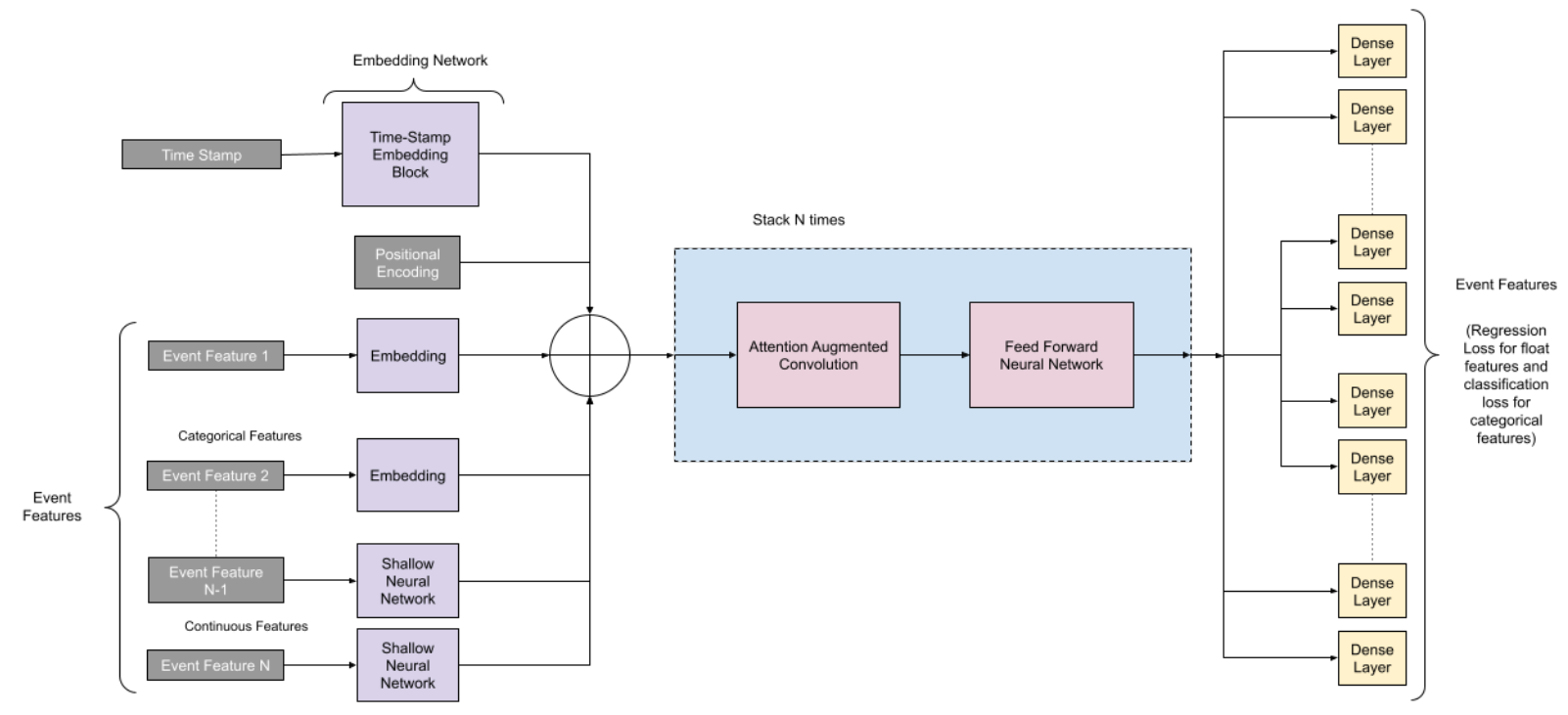

Fig. 2. Our proposed architecture of TabAConvBERT

where Conv denotes convolution and MHA denotes multihead attention.

\section{B. Architecture}

The proposed architecture is shown in Fig. 2 The architecture consists of embedding network, which can encode both categorical inputs (by using simple embedding neural network) and continuous inputs (by using simple shallow neural network). The architecture also consists of a special timestamp embedding block, which is described in more detail in Fig. 3. We first break the original raw timestamp into multiple components such as year, month, day, weekday, week, hour, minute and seconds. These broken up components are discrete and have a finite set of values. For example, month feature has 12 values, week feature has 52 values, weekday feature has 7 values, and so on. Each of these broken up discrete features are passed through an embedding layer. The outputs of each of the embedding layers are then summed-up. Additionally, we also create normalized timestamp features based on dates and time. These float values are then passed through a shallow neural network with "activity regularization" to obtain the vector representations having the same output dimensions of embedding representation. The resulting outputs from these shallow neural networks are also added to the summed-up embedding vector to obtain the final time embedding. The obtained time embedding is then added with input features' embedding and also positional encoding. The resulting summed value is then passed to attention augmented convolution layer and feed forward neural network layer. This combination of attention augmented convolution layer and feed forward neural network layer can be stacked $N$ times to obtain the encoder layer of BERT. Finally, the dense layers in the output part of the network are added to aid pretraining or downstream tasks.

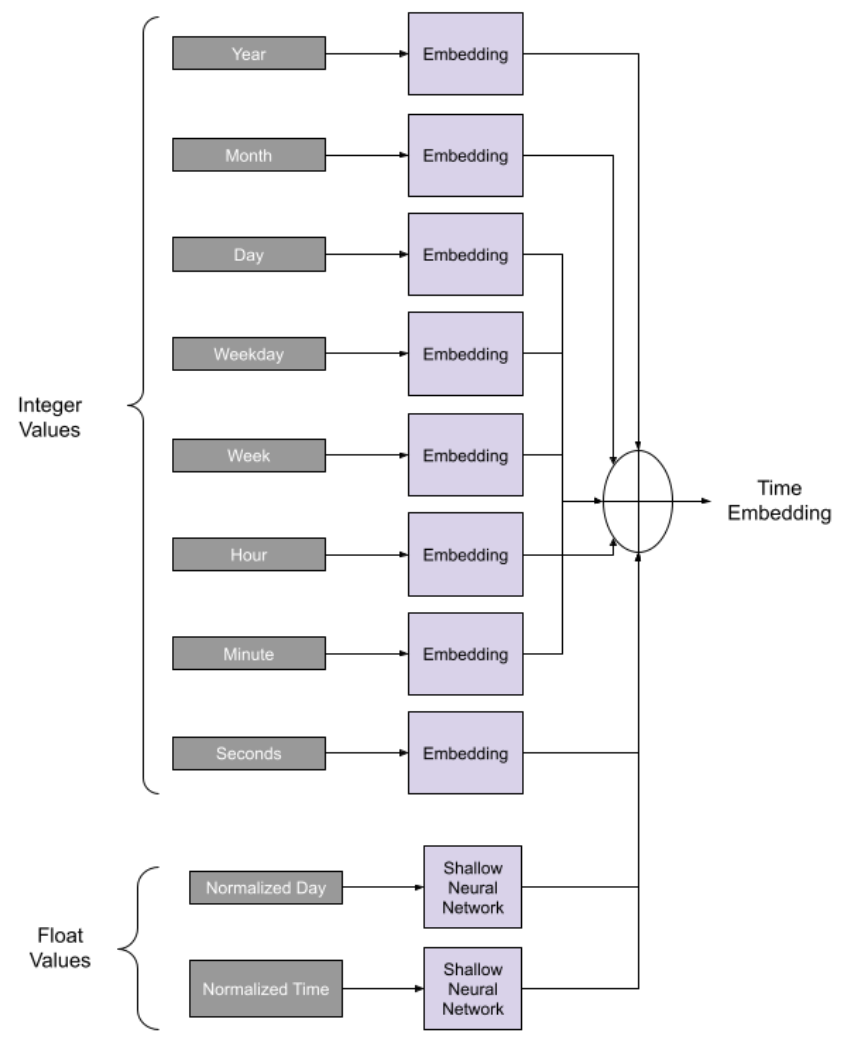

Fig. 3. Our proposed Timestamp Embedding Block

\section{Masking}

For learning representations from the time-series tabular data, we propose a procedure called masked data modeling (MDM), akin to masked language modeling (MLM) employed in NLP. One of the foremost steps in pretraining, as the name suggests, is masking. In MLM, masking is straightforward, since the languages contain only sequence of 

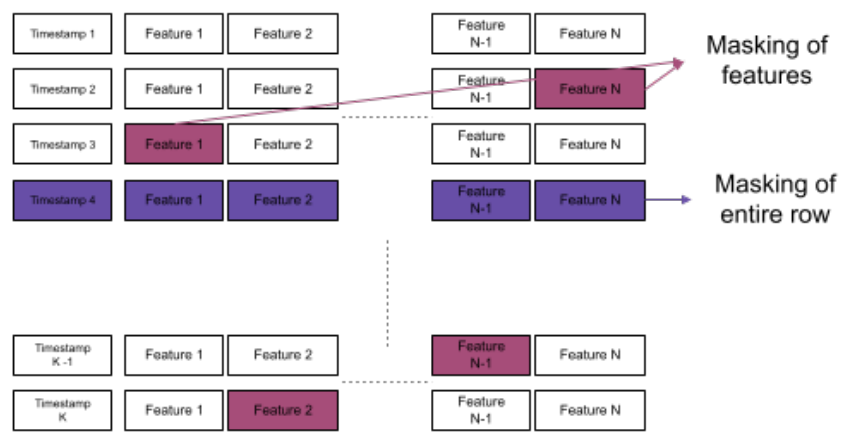

Fig. 4. Our proposed Masking methodology

words. However, in multivariate time-series data, we propose two kinds of masking. For the first kind of masking, we mask out certain percentage of the features at random from the tabular time-series data. This masking is done independently for all the features and is similar to the one performed in [7]. For the second kind of masking, we randomly mask out certain percentage of entire rows of features for the timeseries inputs. Almost all the previous works perform only first kind of masking for the tabular data. But only masking out individual features may not be very effective since often the features are slightly correlated and hence it becomes easier for MDM to predict missing features. The masking of entire row is similar to masking a word in MLM since, in tabular data, the analogue of a "word" is an entire row of features. The figure Fig. 4 gives a clear picture of two types of masking employed in our work.

Additionally, different from other previous works, our framework has the ability to handle continuous type of inputs as is, without resorting to binning. Although for masking the categorical data we can simply have a specific integer token similar to MASK token employed in MLM, for continuous type of inputs, we mask the data by replacing the original values with the mean value of the particular continuous feature.

\section{EXPERIMENTS AND RESUltS}

For our experiments, we employ the dataset provided in the work [1]. The dataset has 24 million transactions from 20,000 users. Each transaction (row) has 12 fields (columns) consisting of both continuous and discrete nominal attributes, such as merchant name, merchant address, transaction amount, etc. For easier comparison, we employ the similar procedures for sampling as performed in [1] by creating samples as sliding windows of 10 transactions, with a stride of 5 . The strategies employed in masking are different since we propose custom masking methodology, however, we perform an experiment by quantizing and creating a vocabulary same as [1]. For masking, we perform $30 \%$ masking of sample's fields and 15\% masking of sample's entire rows. While performing pretraining, we omit the label column to prevent biasing. In our proposed TabAConvBERT architecture, we only employ a single AAConvBERT layer to keep the number of parameters low.

\begin{tabular}{|c|c|}
\hline Method & Fraud F1 Score \\
\hline Baseline MLP [1] & 0.74 \\
\hline Baseline LSTM [1] & 0.73 \\
\hline TabBERT MLP [1] & 0.83 \\
\hline TabBERT LSTM [1] & 0.86 \\
\hline Proposed TabAConvBERT (Only Encoded Data) & 0.888 \\
\hline Proposed TabAConvBERT (With custom masking) & 0.892 \\
\hline Proposed TabAConvBERT & $\mathbf{0 . 8 9 6}$ \\
\hline
\end{tabular}

TABLE I

COMPARISON OF VARIOUS METHODS

For the downstream task of fraud classification, we again perform similar procedures by creating samples by combining 10 contiguous rows (with a stride of 10) in a timedependent manner for each user, and thus obtain 2.4M samples, with 29,342 labeled as fraudulent. For evaluation, we use F1 binary score, on a test set consisting of $480 \mathrm{~K}$ samples for better comparison. For the downstream task, we remove the final dense layers employed during the pretraining stage and a single dense layer for binary classification. For downstream classification task, after pretraining, we freeze all embedding layers and finetune the layers from attention augmented convolution layer. We perform three different kinds of experiments. First, we employ the same quantization and same masking employed in [1], with the main difference being the BERT architecture having attention augmented convolutional layers. Next, we employ our proposed masking scheme but keep the same quantized data. This helps in gauging the improvements offered by our masking scheme. Lastly, we do not perform any encoding and keep all the continuous valued features as is, and also employ timestamp encoding as described in the previous section. Since we employ the same data partitions as described in [1], we directly compare our results from their paper.

As seen from Table II, the proposed methods give significantly improved results on the downstream fraud classification task. The improvements can be seen by directly employing our TabAConvBERT architecture on the quantized or encoded data. The masking procedure also helps in providing a slight improvement in the F1 score. The best results can be seen from our TabAConvBERT with no data preprocessing, i.e., keeping the continuous and categorical values as is and employing timestamp embedding.

\section{CONCLUSION}

In this work, we proposed a novel end-to-end BERT based architecture for time-series tasks. For the first time, we proposed the use of attention augmented convolutions for tabular time-series data and also proposed major modifications to the masking methodology for tabular time-series data. From our experiments, we showed that each of the individual modifications lead to improved results. Our method has a major advantage that it can be directly used with raw data, without resorting to techniques such as feature quantization or encoding. In future, we would like to rigorously evaluate on even larger scale industrial datasets. 


\section{REFERENCES}

[1] I. Padhi, Y. Schiff, I. Melnyk, M. Rigotti, Y. Mroueh, P. Dognin, J. Ross, R. Nair, and E. Altman, "Tabular transformers for modeling multivariate time series," in ICASSP 2021-2021 IEEE International Conference on Acoustics, Speech and Signal Processing (ICASSP). IEEE, 2021, pp. 3565-3569.

[2] T. Mikolov, K. Chen, G. Corrado, and J. Dean, "Efficient estimation of word representations in vector space," arXiv preprint arXiv:1301.3781, 2013.

[3] J. Howard and S. Ruder, "Universal language model fine-tuning for text classification," arXiv preprint arXiv:1801.06146, 2018.

[4] M. E. Peters, M. Neumann, M. Iyyer, M. Gardner, C. Clark, K. Lee, and L. Zettlemoyer, "Deep contextualized word representations," arXiv preprint arXiv:1802.05365, 2018.

[5] A. Vaswani, N. Shazeer, N. Parmar, J. Uszkoreit, L. Jones, A. N. Gomez, L. Kaiser, and I. Polosukhin, "Attention is all you need," arXiv preprint arXiv:1706.03762, 2017.

[6] J. Devlin, M.-W. Chang, K. Lee, and K. Toutanova, "Bert: Pre-training of deep bidirectional transformers for language understanding," arXiv preprint arXiv:1810.04805, 2018.

[7] S. O. Arik and T. Pfister, "Tabnet: Attentive interpretable tabular learning," arXiv preprint arXiv:1908.07442, 2019.

[8] X. Huang, A. Khetan, M. Cvitkovic, and Z. Karnin, "Tabtransformer: Tabular data modeling using contextual embeddings," arXiv preprint arXiv:2012.06678, 2020

[9] W. Dang, B. Zhou, L. Wei, W. Zhang, Z. Yang, and S. Hu, "Tsbert: Time series anomaly detection via pre-training model bert," in International Conference on Computational Science. Springer, 2021, pp. 209-223.

[10] X. Zhang, F. Wei, and M. Zhou, "Hibert: Document level pre-training of hierarchical bidirectional transformers for document summarization," arXiv preprint arXiv:1905.06566, 2019.

[11] R. Pappagari, P. Zelasko, J. Villalba, Y. Carmiel, and N. Dehak, "Hierarchical transformers for long document classification," in 2019 IEEE Automatic Speech Recognition and Understanding Workshop (ASRU). IEEE, 2019, pp. 838-844.

[12] G. Zerveas, S. Jayaraman, D. Patel, A. Bhamidipaty, and C. Eickhoff, "A transformer-based framework for multivariate time series representation learning," in Proceedings of the 27th ACM SIGKDD Conference on Knowledge Discovery \& Data Mining, 2021, pp. 2114-2124.

[13] I. Bello, B. Zoph, A. Vaswani, J. Shlens, and Q. V. Le, "Attention augmented convolutional networks," in Proceedings of the IEEE/CVF international conference on computer vision, 2019, pp. 3286-3295.

[14] S. Bai, J. Z. Kolter, and V. Koltun, "An empirical evaluation of generic convolutional and recurrent networks for sequence modeling," arXiv preprint arXiv:1803.01271, 2018.

[15] J. Hu, L. Shen, and G. Sun, "Squeeze-and-excitation networks," in Proceedings of the IEEE conference on computer vision and pattern recognition, 2018, pp. 7132-7141.

[16] J. Hu, L. Shen, S. Albanie, G. Sun, and A. Vedaldi, "Gather-excite: Exploiting feature context in convolutional neural networks," arXiv preprint arXiv:1810.12348, 2018.

[17] J. Park, S. Woo, J.-Y. Lee, and I. S. Kweon, "Bam: Bottleneck attention module," arXiv preprint arXiv:1807.06514, 2018.

[18] S. Woo, J. Park, J.-Y. Lee, and I. S. Kweon, "Cbam: Convolutional block attention module," in Proceedings of the European conference on computer vision (ECCV), 2018, pp. 3-19. 